\title{
RUTHENIUM(II) COMPLEXES WITH CYTOTOXIC ACTIVITY EMBEDDED IN HYDROXYPROPYL METHYLCELLULOSE/SODIUM ALGINATE MUCOADHESIVE HYDROGELS
}

\author{
MIRELA-FERNANDA ZALTARIOV, BIANCA-IULIA CIUBOTARU, LILIANA VEREȘTIUC, \\ DRAGOȘ PEPTANARIU, ${ }^{* *}$ DOINA MACOCINSCHI ${ }^{* * *}$ and DANIELA FILIP ${ }^{* * * *}$ \\ Department of Inorganic Polymers, "Petru Poni” Institute of Macromolecular Chemistry, \\ 41A, Gr. Ghica-Voda Alley, 700487, Iasi, Romania \\ "Department of Biomedical Sciences, Faculty of Medical Bioengineering, Grigore T. Popa University of \\ Medicine and Pharmacy, 9-13 Kogalniceanu Str., 700454, Iasi, Romania \\ ** Center of Advanced Research in Bionanocojugates and Biopolymers, "Petru Poni" Institute of \\ Macromolecular Chemistry, 41A, Gr. Ghica-Voda Alley, 70487, Iasi, Romania \\ *** Department of Polyaddition and Photochemistry, "Petru Poni" Institute of Macromolecular Chemistry, \\ 41A, Gr. Ghica-Voda Alley, 700487, Iasi, Romania \\ \Corresponding author: M.-F.Zaltariov, zaltariov.mirela@icmpp.ro
}

Dedicated to the $70^{\text {th }}$ anniversary of the Department of Pulp and Paper, "Cristofor Simionescu" Faculty of Chemical Engineering and Environmental Protection, "Gheorghe Asachi” Technical University of Iasi

\begin{abstract}
Mucoadhesive hydrogels based on hydroxypropylmethyl cellulose/sodium alginate have been prepared and used as matrix for encapsulation and delivery of new ruthenium(II) complexes with selective cytotoxic activity on human melanoma (MEWO) cells. The structure of the hydrogels was investigated by FT-IR spectroscopy and SEM microscopy. The encapsulation and the delivery of ruthenium(II) complexes from polymeric matrices were monitored by UV-vis spectroscopy. Thermal and moisture stabilities of the composite gels were evidenced by FT-IR and Dynamic Vapor Sorption (DVS) analyses. The mucoadhesion properties of the hydrogels have been investigated on bladder mucosa, the most mucoadhesive gel being selected as a promising precursor composite for anticancer drug delivery systems.
\end{abstract}

Keywords: hydroxypropyl methylcellulose, sodium alginate, mucoadhesive hydrogels, metal complexes, cytotoxicity

\section{INTRODUCTION}

The development of drug delivery systems has gained increasing attention by the use of biodegradable polymers as carriers with targeted effects. A lot of polysaccharides, such as chitosan, sodium alginate, hydroxypropylmethyl cellulose, agar, guar or gellan gum, have been applied in different formulations as smart materials (controlled release depending on the volume changes, development of viscous protective layer barriers against water influx and drug efflux in aqueous media). ${ }^{1}$ Among other polymers, sodium alginate, with its unique property of gel formation in the presence of cations in aqueous media, and HPMC, with its hydrophobicity, are good candidates for the preparation of drug delivery systems as an alternative strategy to create new platforms, with enhanced therapeutic and less toxic side effects of drugs. Natural polymers meet the requirements necessary for improved drug formulations, such as biodegradability and biocompatibility, which are often difficult to achieve for synthetic polymers. ${ }^{2}$ Cellulose and its 
functionalized derivatives are the most promising candidates for preparing drug delivery systems that could exhibit stimuli-response properties, amphiphilic behavior or increased solubility in aqueous media of poorly soluble compounds (drugs). ${ }^{3}$ Low solubility is a major problem for most organic drugs. A promising approach to overcome the solubility limitation consists in embedding drugs in polymeric blends, which then release a therapeutic dosage of the drug at target sites. Several formulations based on adequate precursors - organic, inorganic or hybrid materials - have been designed. Thus, interpolymeric complexes based on alginate and chitosan proved to be efficient as carriers for Nystatin in mucosal delivery, with a moderate drug loading capacity of $15 \%$, but sufficient to have a therapeutic effect onto the skin surface. ${ }^{4}$ In another approach, carboxymethyl cellulose acetate (CMCA) and carboxymethyl cellulose acetate butyrate (CMCAB) have been used as stabilizers of sulfadiazine drug in water dispersion, with a higher drug loading capacity of $42.88 \%$, due to the presence of hydropbobic moieties in the polymeric composites. ${ }^{5}$ Recent studies reported on the use of HPMC as tablet matrix forming polymer and fumaric acid at acidic micro-environmental $\mathrm{pH}$ to develop sustained release of dipyridamole. ${ }^{6}$ In other studies, hybrid composites based on natural polymers, such as sodium alginate/starch, have been used as adsorbents for metal ions removal, especially copper(II) ${ }^{7}$, while nanofibers electrospun from cellulose acetate (CA) and ethyl cellulose (EC) polymer blends have been used as water insoluble microparticle drug delivery systems. ${ }^{8}$ Particularly, metal complexes based on organic ligands are often poorly water soluble, which makes antiproliferative screening difficult and reduces the bioavailability of the potential drugs in the human body. To prevent this disadvantage, some researchers used cellulose nanowhiskers to prepare a macromolecular support to assist the fabrication of some palladium(II) complexes with antitumor activity on human colon carcinoma cell line HCT116. The complex loaded cellulose nanoparticle composite proved to be a very effective prodrug system with an inhibitory concentration in the micromolar range $(203 \mu \mathrm{g} / \mathrm{mL}$ after $24 \mathrm{~h}){ }^{2}$

Referring to ruthenium(II) complexes, a large variety of polymers, lipids or mixed organicbased materials are used to facilitate their delivery, by improving solubility and reducing toxicity, in order to increase their selectivity on a targeted tumor cell line and to achieve a therapeutic effect. Ruthenium complexes are a good alternative to platinum derivatives, which are known for their toxic side effects, development of drug resistance, a lack of selectivity on tumor/normal cell lines and an unselective mode of action. Among other anticancer drugs, two ruthenium(II) complexes (NAMI-A and KP 1019) have entered phase II clinical trials with increased selectivity and a higher effect on metastatic tumors. Varied drug release carriers obtained by chemical conjugation and physical encapsulation have been reported for ruthenium complexes. Thus, poly(lactic acid), mesoporous silica, metal-organic frameworks (MOFs), amphiphilic nucleoside derivatives based on lipid residues of oleoyl or cholesteroxyacetyl compounds and hydrophilic oligo(ethylene glycol) have been used as carriers for their controlled release. More than that, such conjugate systems proved higher anticancer activity, increased aqueous stability and lower toxicity.

Herein, we report on the preparation of new hydrogel formulations based on HPMC/SA blends, with enhanced mucoadhesivity on bladder mucosa, and their use for loading and release of new ruthenium(II) complexes. This is the first report on the application of natural polysaccharide composites based on HPMC in embedding and delivery of ruthenium(II) complexes with selective cytotoxic activity on human melanoma (MEWO) cells.

\section{EXPERIMENTAL}

\section{Materials}

Anise oil was extracted according to the procedure described by J. Lawless. ${ }^{10}$ Hydroxypropyl methylcellulose (HPMC) (Aldrich) and sodium alginate (SA) (Aldrich) were used as received for obtaining gel formulations by their dispersion in small quantities of distilled water to normal hydration. The composition of the gel formulations is shown in Table 1. Ruthenium(II) complexes (MZ08 and MZ13) have been prepared as described in an earlier study. ${ }^{11}$ Their structure can be seen in Scheme 1.

A dialysis tubing cellulose membrane was purchased from Aldrich. Bladder mucosa was obtained from the Training and Research Center in Tissue Engineering, Artificial Organs and Regenerative Medicine, Faculty of Medical Bioengineering, Grigore T. Popa University of Medicine and Pharmacy, Iasi, Romania Iasi.

Human melanoma (MEWO) cell line was purchased from CLS Cell Lines Service $\mathrm{GmbH}$, 
Germany, while Normal Human Dermal Fibroblasts (NHDF) cell line from PromoCell. All the materials needed for MTS cytotoxicity tests: Eagle's Minimal Essential Medium alpha (aMEM), Dulbecco's Modified Eagle Medium without phenol red (DMEM), $1 \%$ Penicillin-Streptomycin-Amphotericin B mixture,
Fetal Bovine Serum (FBS), Trypsin-Versene mixture from Lonza (EDTA) were purchased from Biochrom GmbH, Germany. The Cell Proliferation Assay and Tryple test were received from Promega and Thermo Fisher Scientific.

Table 1

Formulations of the mucoadhesive gels (V-VIII)

\begin{tabular}{lcccc}
\hline Composition, g & V & VI & VII & VIII \\
\hline HPMC & 0.1 & 0.2 & 0.3 & 0.4 \\
Sodium alginate & 3 & 3 & 3 & 3 \\
Distilled water q.s. & 100 & 100 & 100 & 100 \\
Anise oil & 0.15 & 0.15 & 0.15 & 0.15 \\
\hline
\end{tabular}
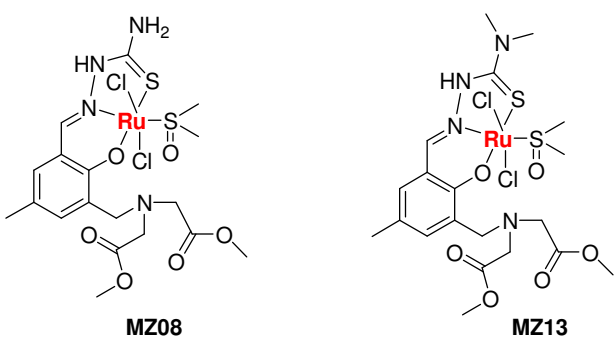

Scheme 1: Chemical structure of ruthenium(II) complexes

\section{Methods}

Fourier transform infrared (FT-IR) spectroscopy

Infrared spectra were recorded in the ATR (Attenuated Total Reflectance) mode on a Bruker Vertex 70 spectrometer from Bruker Optics, Germany. A spectral range of $4000-600 \mathrm{~cm}^{-1}$ and a resolution of $4 \mathrm{~cm}^{-1}$ were established for the experimental set-up.

The stability of the gels in the temperature range between 25 and $55{ }^{\circ} \mathrm{C}$ was evaluated by FT-IR spectroscopy. The samples were deposited on the surface of a diamond crystal of the ATR-FTIR accessory, equipped with a temperature controller, and the temperature was varied with $10{ }^{\circ} \mathrm{C}$ at each recording.

\section{Dynamic vapor sorption (DVS) analysis}

The moisture stability of the gels in the $0-90 \%$ relative humidity range $(\mathrm{RH})$ was investigated by a gravimetric analyzer (IGAsorp, Hiden Analytical, Warrington, UK), equipped with an ultrasensitive microbalance. Sorption-desorption isotherms were registered during the experiments and the sorption capacity of each sample was determined. Before the sorption measurements, all the samples were dried in flowing nitrogen $(250 \mathrm{~mL} / \mathrm{min})$ at $25^{\circ} \mathrm{C}$.

\section{Scanning electron microscopy (SEM) analysis}

The surface morphology of the unmetallized lyophilized ( $48 \mathrm{~h}$ at $-57{ }^{\circ} \mathrm{C}$ and 0.045 mbar) hydrogel samples before and after embedding ruthenium(II) complexes was investigated with an ESEM Quanta 200
Scanning Electron Microscope (Netherlands), operating at $20 \mathrm{kV}$ in the low vacuum mode with an integrated EDS system, GENESIS XM 2i EDAX with SUTW detector, the magnification being indicated on the micrographs.

\section{Mucoadhesion tests}

The mucoadhesion properties were evaluated by a TA.XT Plus Texture apparatus (Stable Micro Systems, UK). Before the tests, the samples were cut to a standard size to allow dropping on the support (a mobile cylinder component of the equipment). Bladder tissue and a cellulose membrane having a surface of 4 $\mathrm{cm}^{2}$ were placed in the sample device. During the recordings, the cylindrical holder with the pieces of samples was lowered with $1 \mathrm{~mm} / \mathrm{s}$. The contact force and the contact time were established at $1 \mathrm{gF}$ and $30 \mathrm{~s}$, respectively.

\section{MTS assay}

Normal (NHDF) and cancer cells (MEWO) were cultivated at $37{ }^{\circ} \mathrm{C}+5 \% \mathrm{CO}_{2}$ humidity, in tissue culture flasks containing a medium made of aMEM, $10 \%$ FBS and $1 \%$ streptomycin-penicillinamphotericin. Tryple test was used for NHDF cells and Trypsin-Versene for MEWO cells. Ru(II) complexes were dissolved in Millipore water and used at different concentrations in DMEM. The incubation time for cells containing $\mathrm{Ru}$ (II) complexes was $72 \mathrm{~h}$. The iMark plate reader was used to read the absorbance at 490 $\mathrm{nm}$, characteristic of the formazan obtained by 
mitochondrial activity. The relative cell viability was calculated with the formula: $\mathrm{V}=100 \mathrm{x}(\mathrm{S}-\mathrm{B}) /(\mathrm{C}-\mathrm{B})$, where $\mathrm{V}$ is relative viability, $\mathrm{S}$ is the absorbance of the sample, B is the absorbance of the blank, C is the absorbance of the control sample (cell medium without $\mathrm{Ru}(\mathrm{II})$ complex).

\section{Encapsulation of ruthenium(II) complexes in HPMC/SA gels}

UV-vis spectroscopy (Specord 200 Spectrophotometer) was used to evaluate the encapsulation of the metal complexes in HPMC/SA gels. Before embedding in the gels, the $\mathrm{Ru}(\mathrm{II})$ complexes were dissolved in ethanol/Millipore water (4:1), at a concentration of $5.8 \times 10^{-4} \mathrm{M}$, and the solid gels (about $70 \mathrm{mg}$ ) were added to this solution. After 2 $\mathrm{h}$, the concentration of the residual solution of $\mathrm{Ru}(\mathrm{II})$ complexes was measured and a loading capacity of $58 \%$ was calculated. After encapsulation, the gels were washed with ethanol and dried in vacuum for $24 \mathrm{~h}$. The delivery of $\mathrm{Ru}(\mathrm{II})$ complexes from the HPMC/SA gels was monitored by UV-vis spectroscopy (absorbance at $360 \mathrm{~nm}$ (MZ08) and $320 \mathrm{~nm}$ (MZ13), assigned to LMCT (ligand-to-metal charge transfer) and MLCT (metal-to-ligand charge transfer)) in PBS pH 7.4 at 37 ${ }^{\circ} \mathrm{C}$.

\section{RESULTS AND DISCUSSION FT-IR spectroscopy}

Infrared spectra of the HPMC/SA hydrogels showed the characteristic vibrations assigned to the hydroxyl groups in the 4000-3000 $\mathrm{cm}^{-1}$ spectral range. Other specific bands are those assigned to the asymmetric and symmetric stretching vibrations of the carboxylate groups at $1600 \mathrm{~cm}^{-1}$ and $1410 \mathrm{~cm}^{-1}$, respectively, and those specific to the components of the polymeric system, similar to the HPMC/PAA hydrogels previously described. ${ }^{12}$

By using the second derivative of the spectra, the position of the maxima of the hydroxyl bands was established. The deconvolution of the spectra was realized by the curve-fitting method, using OPUS software (Fig. 1).

The $\mathrm{H}$ bonding distances in the HPMC/SA gels were obtained by using the Sederholm equations ${ }^{13}$ and the energy of the $\mathrm{H}$ bonds $\left(\mathrm{E}_{\mathrm{H}}\right)$ (Table 2) was calculated according to $\mathrm{H}$. Struszczyk. ${ }^{14}$
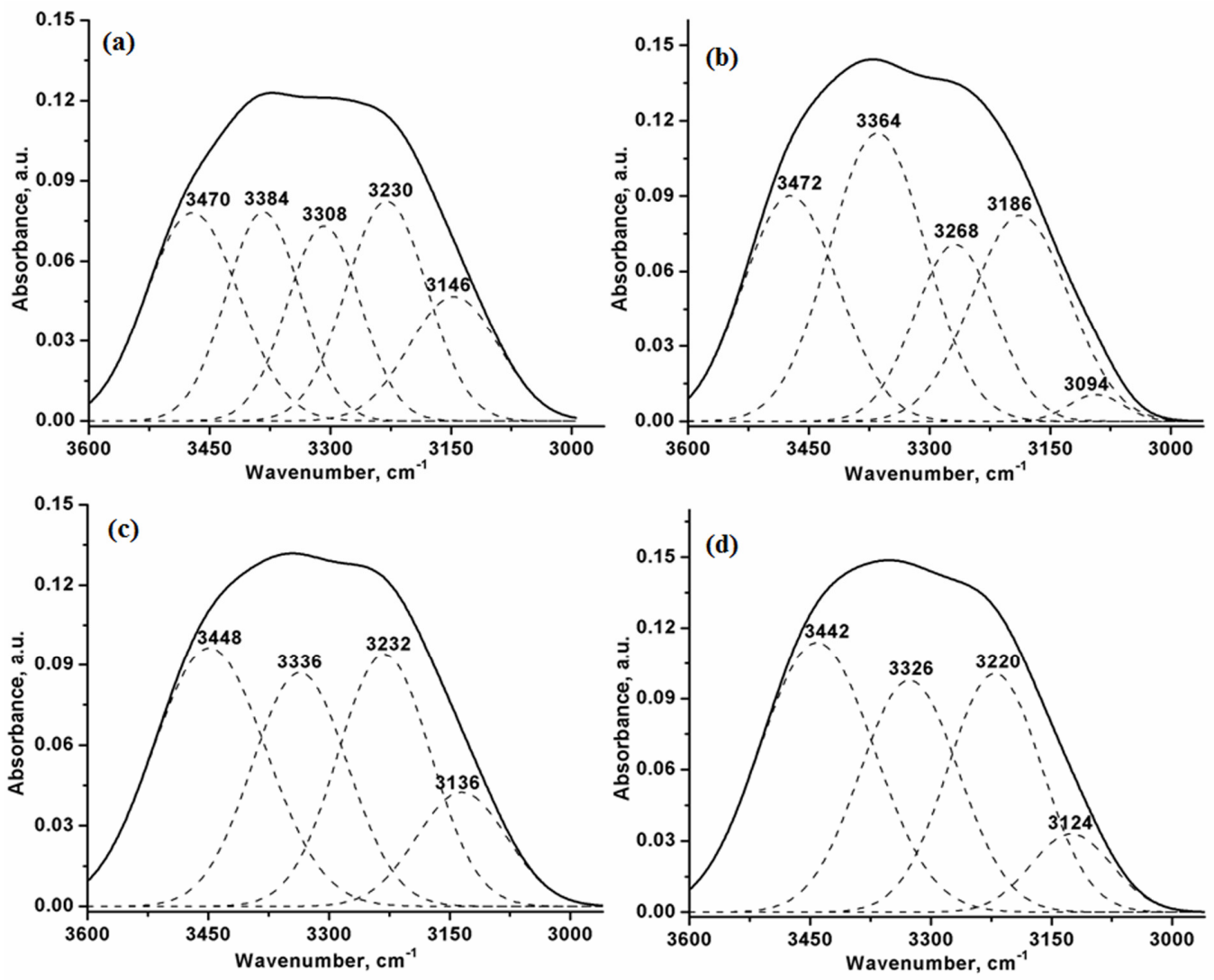

Figure 1: Deconvoluted IR spectra of HPMC/SA based hydrogels in the $3600-3000 \mathrm{~cm}^{-1}$ spectral range: (a) V, (b) VI, (c) VII, and (d) VIII 
Table 2

Curve-fitting results of the -O-H spectral region, energy and distances of the hydrogen bonds of studied hydrogels

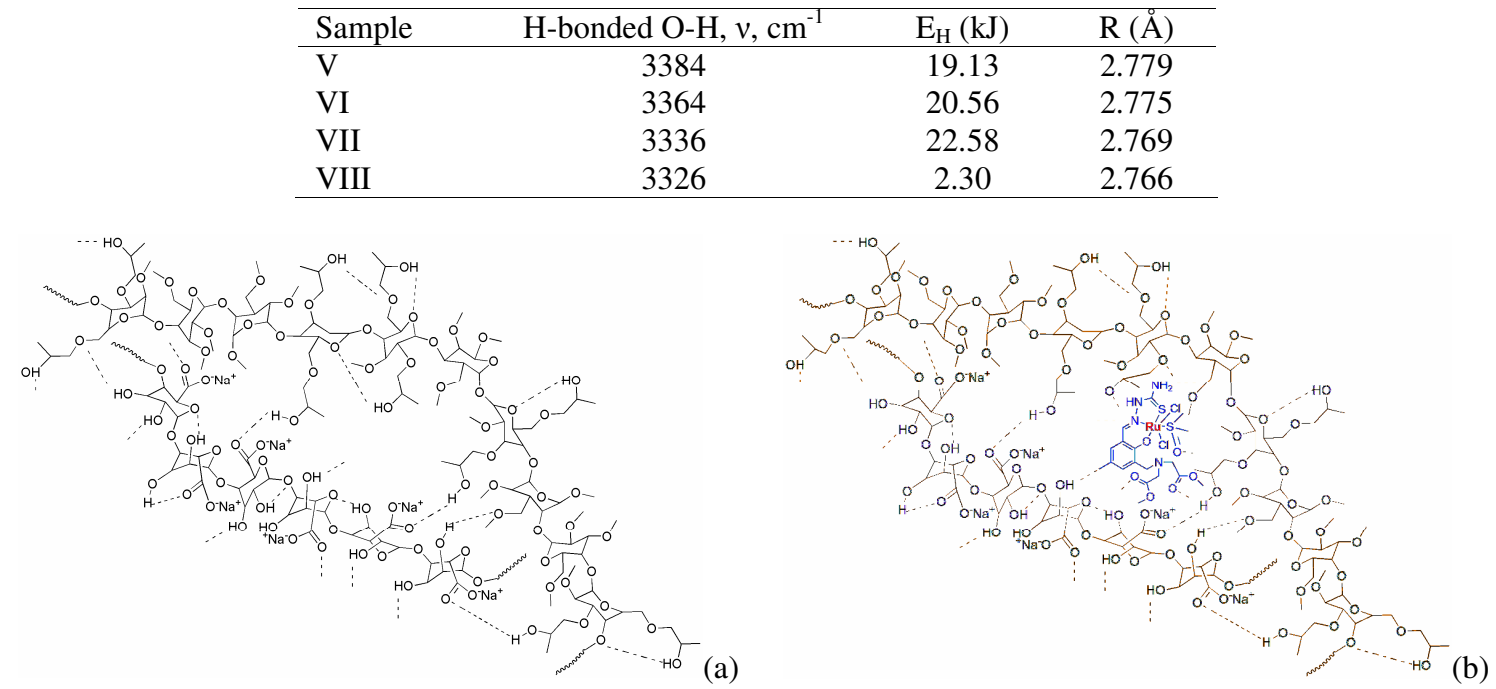

Scheme 2: Structural representation of HPMC/SA hydrogels (a) and of embedded Ru(II) complex (MZ08)

in the HPMC/SA gel

The H-bonding interactions between HPMC and SA were found to be weaker, compared to those of HPMC/PAA hydrogels ${ }^{12}\left(\mathrm{E}_{\mathrm{H}}=19.13 \mathrm{~kJ}\right)$, but also they increase with the HPMC content in the mixture $\left(E_{\mathrm{H}}=23.30 \mathrm{~kJ}\right)$. This could be explained by the type of network interactions, which particularly occur in this case between the carbonyl group as acceptor and the $\mathrm{OH}$ groups from HPMC as donor.

The characteristic $-\mathrm{OH}$ vibrations for $\mathrm{H}$ bonds intermolecular interactions between the polymeric chains (HPMC and SA) occur at $3384 \mathrm{~cm}^{-1}$ (V), $3364 \mathrm{~cm}^{-1}$ (VI), $3336 \mathrm{~cm}^{-1}$ (VII) and $3326 \mathrm{~cm}^{-1}$ (VIII), while those assigned to the $\mathrm{H}$ bonding intramolecular interactions are blueshifted by 28 $\mathrm{cm}^{-1}$ upon increasing the HPMC content. The other $-\mathrm{OH}$ vibrations in the deconvoluted spectra are assigned to the intramolecular -O-H...C-O-C and $-\mathrm{O}-\mathrm{H} \ldots \mathrm{O}(6)$ interactions in the structure of HPMC and SA, and are also blueshifted upon increasing the content of HPMC (Fig. 1). ${ }^{15}$ These interactions led to the formation of highly connected networks (Scheme 2).

The stability of the gels during the heating/cooling cycle was also studied by FT-IR spectroscopy. The dynamic character of the $\mathrm{H}$ bond formation could be observed: by increasing the temperature from 27 to $55{ }^{\circ} \mathrm{C}$, the appearance of new bands at $3700 \mathrm{~cm}^{-1}$ and $1700 \mathrm{~cm}^{-1}$ is noted, which are specific to non-bonded $\mathrm{OH}$ and carboxylate groups (Fig. 2). The area corresponding to these bands also increases by increasing the HPMC content in the gels. Upon cooling, the reformation of $\mathrm{H}$-bonds occurs with a lower speed, owing to the flexibility of the polymeric chains during the heating process, which cannot reach a highly ordered structural motif (Fig. 2).

The successfully completed embedding of $\mathrm{Ru}(\mathrm{II})$ complexes into the HPMC/SA hydrogels was proved initially by IR spectroscopy (Fig. 3).

The main changes occurring in the IR spectra of VII-MZ08 are due to the interactions between the $\mathrm{Ru}$ (II) complex and the polymeric gel. One can observe the redshifts of the ester band assigned to the $\mathrm{Ru}(\mathrm{II})$ complex and of the carboxylate band in the structure of SA by $7 \mathrm{~cm}^{-1}$ in the polymeric matrix. Also, the characteristic $\mathrm{OH}$ bands in the embedded gel are redshifted by $50 \mathrm{~cm}^{-1}$, as compared to the HPMC/SA gel (VII), suggesting a highly connected network between the metal complex and the polymeric matrix. Also, the bands at $1259 \mathrm{~cm}^{-1}$ and $1213 \mathrm{~cm}^{-1}$ in the IR spectrum of VII-MZ08 are assigned to the thioamide bands present in the $\mathrm{Ru}(\mathrm{II})$ complex (Fig. 3). 

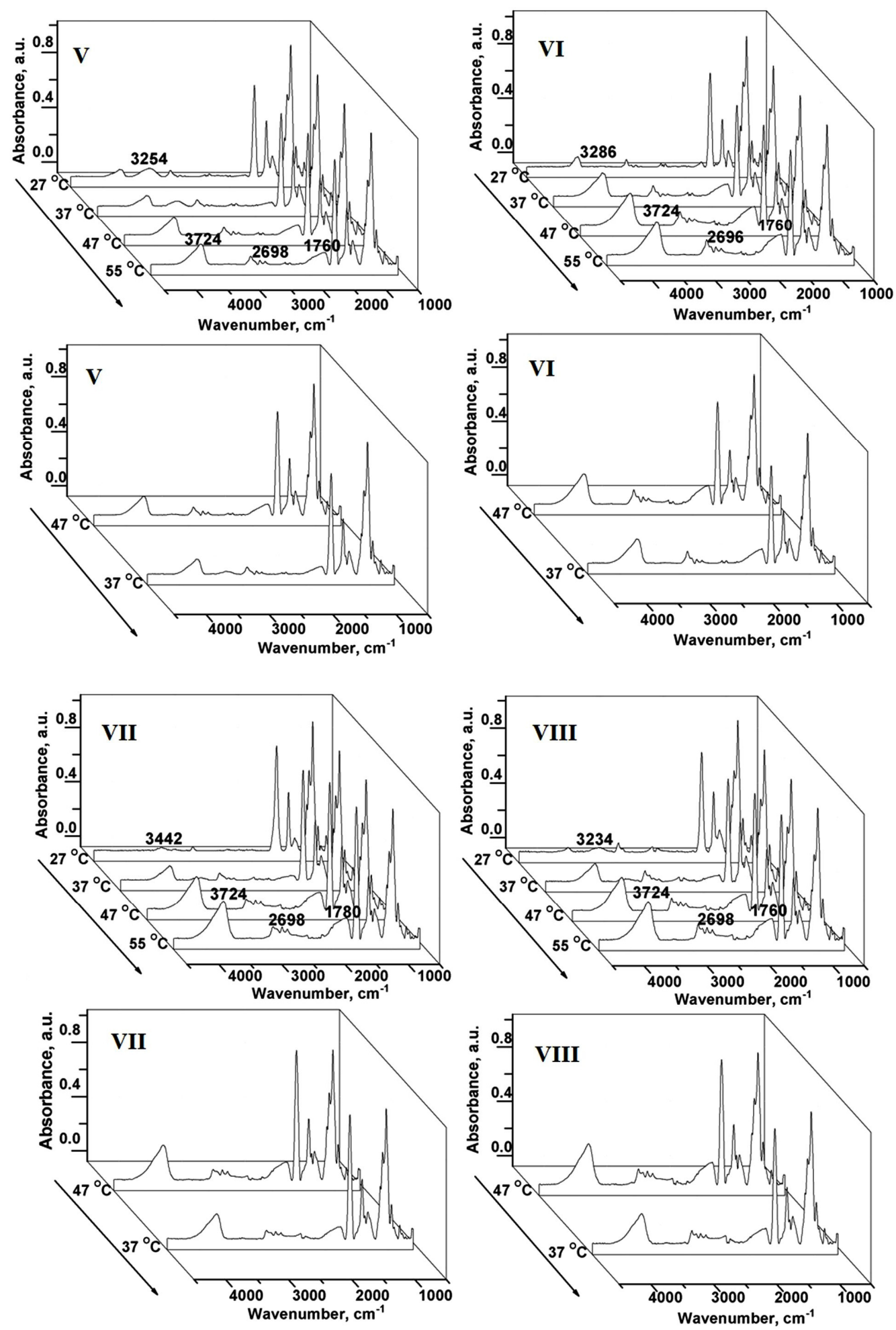

Figure 2: ATR-FTIR spectra of hydrogels V, VI, VII and VIII during the heating from 27 to $55^{\circ} \mathrm{C}$ and cooling process 


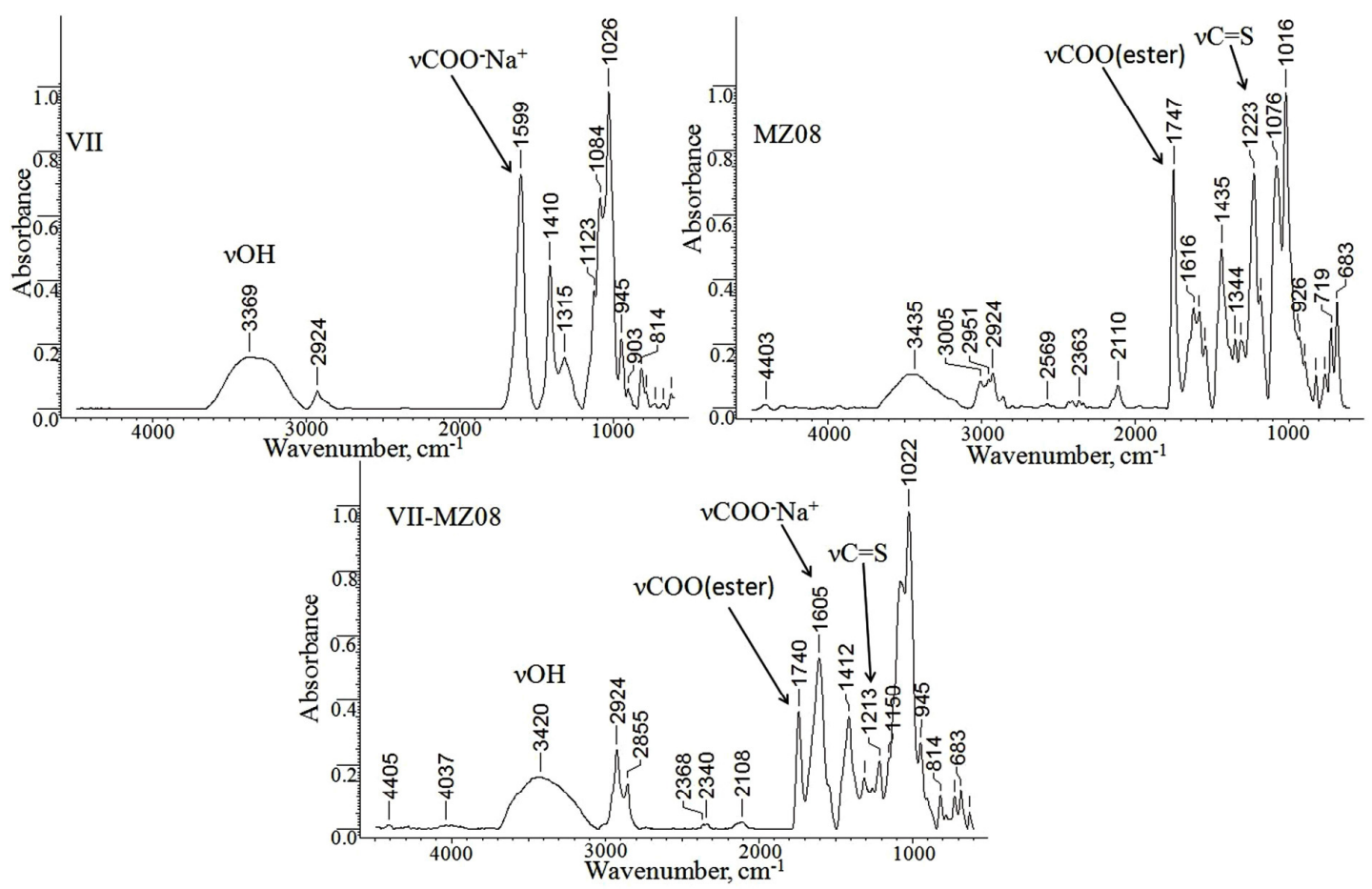

Figure 3: ATR-FTIR spectra of HPMC/SA hydrogel (VII), HPMC/SA hydrogel with encapsulated Ru(II) complex (VII-MZ08) and of Ru(II) complex MZ08

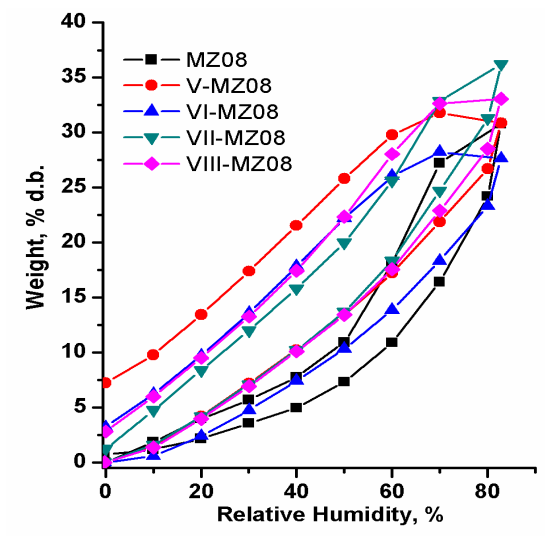

Figure 4: Sorption/desorption isotherms of MZ08 and embedded HPMC/SA gels

\section{DVS analysis}

The moisture stability of $\mathrm{Ru}(\mathrm{II})$ complexes, particularly MZ08, embedded in HPMC/SA gels was evaluated by sorption/desorption isotherms (Fig. 4). In all the gels with embedded $\mathrm{Ru}$ (II) complexes, similar shapes and sorption capacity values were found, suggesting hydrophilic materials with enhanced hydrophilic behavior at higher RH. MZ08 showed a typical Type IV water sorption isotherm, with an increased water uptake at lower humidity, and additionally adsorbed multilayer water due to the presence of hydrophilic sites (i.e. ester, $\mathrm{NH}, \mathrm{NH}_{2}$ groups). The maximal sorption capacity for MZ08 was $30.78 \%$. The $\mathrm{Ru}(\mathrm{II})$ embedded HPMC/SA gels with different HPMC content are characterized by the same Type IV sorption isotherms, but the sorption uptake at lower RH is higher than that of MZ08 and does not change by increasing the HPMC content. The maximal sorption capacity of $\mathrm{Ru}(\mathrm{II})$ embedded gels is $30.85 \%$ for V-MZ08, $27.63 \%$ for VI-MZ08, 36.22\% for VII-MZ08 and 33.07\% 
for VIII-MZ08, respectively. One can also observe the good moisture stability of MZ08 and the retention of about $3-7 \%$ of water in the case of the embedded gels, due to the re-adsorbed water during the desorption process. ${ }^{16}$

SEM images revealed alteration of the morphology of the superporous and highly interconnected hydrogel networks (i.e. VII and VIII) (Fig. 5), as compared to the $\mathrm{Ru}$ (II) embedded gels (VII-MZ08 and VIII-MZ13). This aspect evidenced that $\mathrm{Ru}(\mathrm{II})$ complexes were encapsulated in the gel pores. By increasing the HPMC content, the ordered porous structure (i.e. VII) became irregular, with non-uniform porosity, because of the stronger intermolecular interactions. In the large pores, aggregates of the $\mathrm{Ru}$ (II) complex can be observed (VIII-MZ13). The porosity of the gels will influence both the loading capacity and the release behavior.

\section{Mucoadhesion test}

The adhesion properties of all the HPMC/SA gels were evaluated before incorporation of $\mathrm{Ru}(\mathrm{II})$ complexes in order to select the most adhesive compound for drug delivery studies. All the hydrogels were tested on a dialysis cellulose membrane (Fig. 6a) and bladder mucosa (Fig. 6b) at $\mathrm{pH} 5$ and $37{ }^{\circ} \mathrm{C}$. The selection of bladder mucosa is due to recent reports regarding the application of the $\mathrm{Ru}(\mathrm{II})$-polypyridyl compound (TLD-1433) in patients with bladder cancer (phase IB clinical trials in 2015). ${ }^{17}$

One can observe an increased detachment force and work of adhesion by increasing the HPMC content in the gels, due to complex intermolecular interaction between the gels and the cellulose membrane or bladder mucosa.

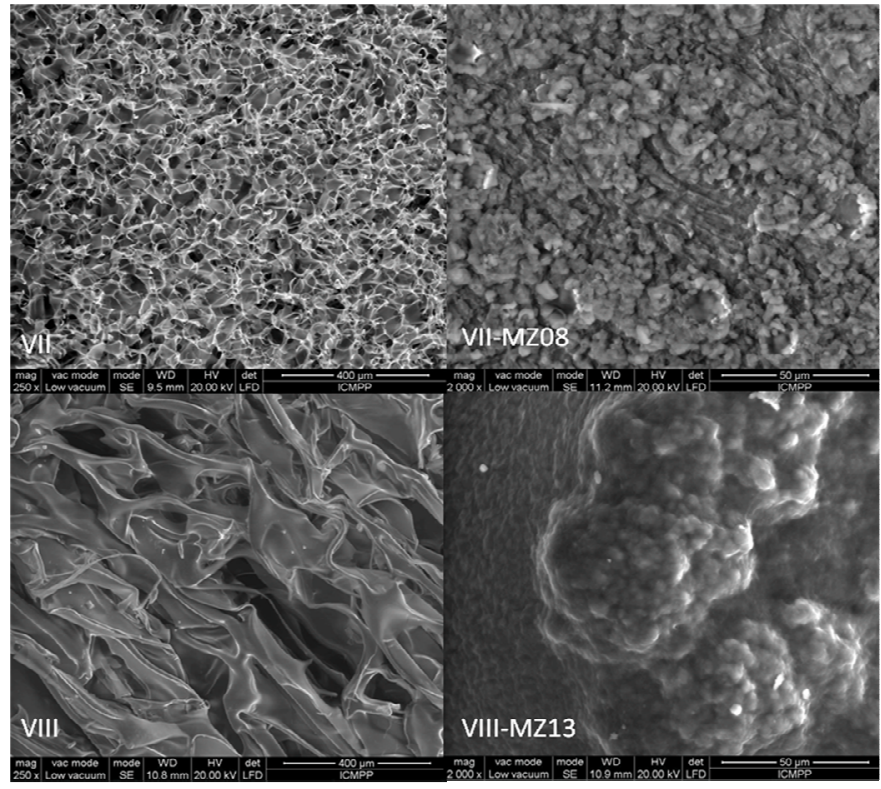

Figure 5: SEM images of HPMC/SA gels before and after encapsulation of Ru(II) complexes
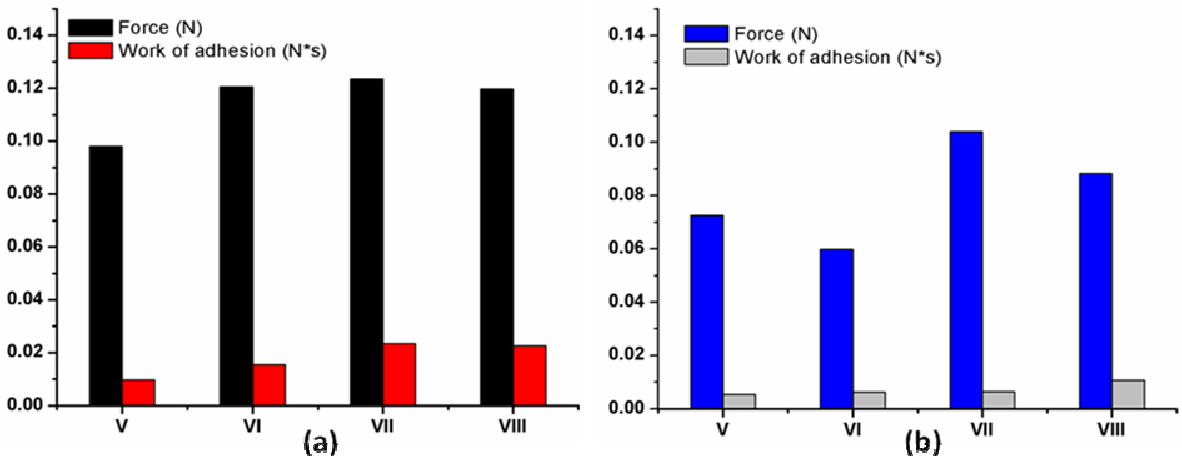

Figure 6: Bioadhesion (a) and mucoadhesion (b) results of HPMC/SA gels 


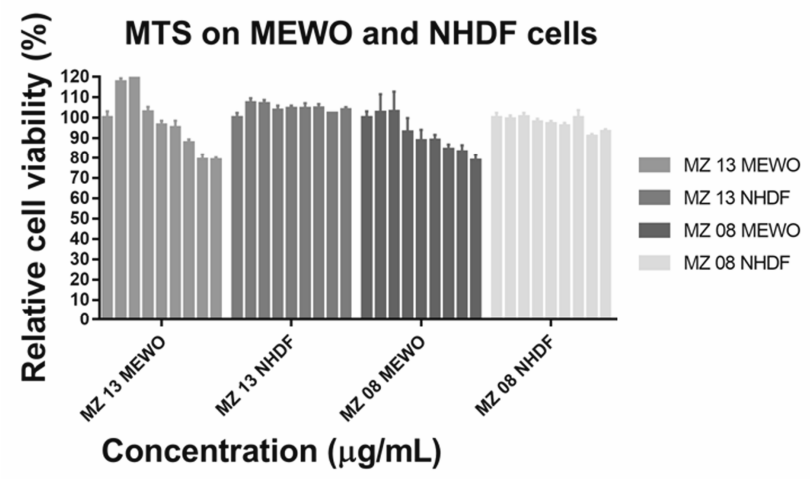

Figure 7: Cytotoxicity of MZ08 and MZ13 on MEWO and NHDF
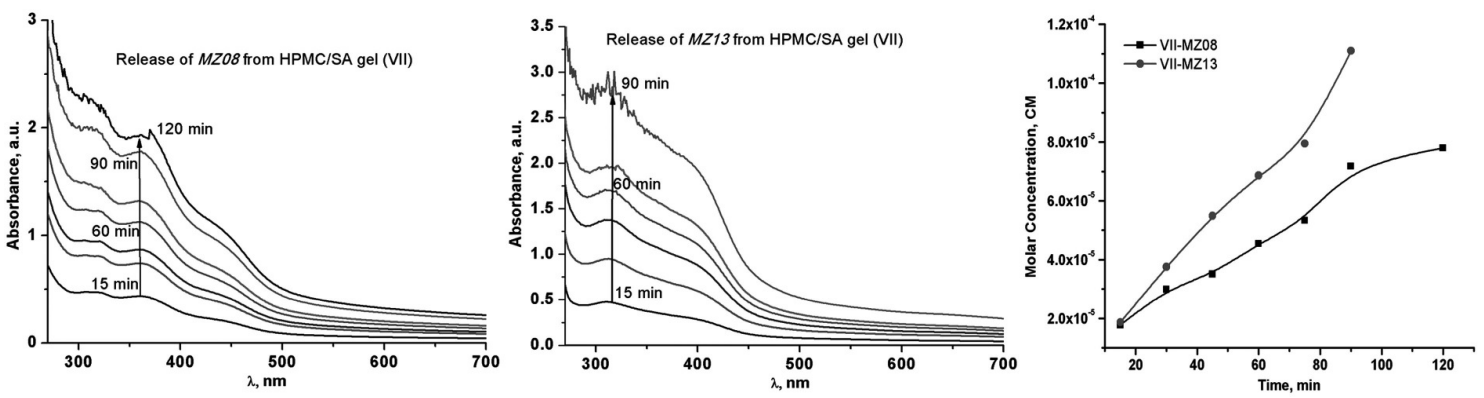

Figure 8: UV-vis spectra during the delivery of MZ08 and MZ13 and the delivery curve of the studied complexes from HPMC/SA gel (VII)

On the cellulose membrane, a higher detachment force was observed for hydrogels VII and VIII (Fig. 6a), while on bladder mucosa, the most adhesive was VII (Fig. 6b). The chemical composition and structural morphology, such as different porosity (Fig. 5), of these gels could explain their different adhesiveness, even if the HPMC content in VIII is higher than in VII. The uniformity in terms of the size and distribution of pores (i.e. in VII) will allow easy intermolecular interactions with mucosa. We should mention that the work of adhesion increased with the HPMC content, when the gels were tested on the cellulose membrane, while it remained relatively constant on bladder mucosa, suggesting that different mechanisms are involved in the adhesion properties of these materials.

Based on these results, we selected gel VII for loading and delivery of $\mathrm{Ru}(\mathrm{II})$ complexes.

\section{MTS assay}

The cytotoxicity of the $\mathrm{Ru}(\mathrm{II})$ complexes was evaluated on MEWO (human melanoma) and NHDF (normal cells), by measuring the mitochondrial reductase activity. We chose human melanoma cells to test our $\mathrm{Ru}(\mathrm{II})$ complexes because melanoma is an aggressive and highly metastatic disease, ${ }^{18}$ and we wanted to find a suitable product with selectivity for this cancer cell line. $\mathrm{Ru}(\mathrm{II})$ complexes MZ08 and MZ13 proved to be biocompatible, the cell viability on NHDF cells being over $100 \%$ at all the tested concentrations $(70-250 \mu \mathrm{g} / \mathrm{mL})$. Their cytotoxicity against MEWO cells was relatively weak, even if the concentration of $\mathrm{Ru}$ (II) complexes was increased five times. Some selectivity could be observed at higher concentrations $>200 \mu \mathrm{g} / \mathrm{mL}$ (Fig. 7), with a cell viability of about $80 \%$ for both complexes MZ08 and MZ13. These results are similar to the findings of other studies, where ruthenium complexes showed selectivity, especially on metastasis. ${ }^{18}$

\section{Delivery of Ru(II) complexes from HPMC/SA gel (VII)}

The delivery of $\mathrm{Ru}(\mathrm{II})$ complexes from VII was evaluated by UV-vis spectroscopy in PBS at pH 7.4 and $37{ }^{\circ} \mathrm{C}$ (Fig. 8). The selection of the HPMC/SA gel VII was made on the basis of 
mucoadhesion properties. One can observe a fast delivery process, determined by the hydrophilic character of the complexes and gel VII, which decomposes easily, the main forces supporting the network being $\mathrm{H}$-bonds. The advantage of the fast delivery of $\mathrm{Ru}$ (II) complexes is the accumulation of a high concentration of the drug for achieving a therapeutic effect, as the MTS study revealed.

\section{CONCLUSION}

New mucoadhesive hydrogel formulations based on HPMC/SA blends were prepared. Their structure was confirmed by FT-IR spectroscopy, where strong intermolecular hydrogen bonding interactions between HPMC and SA were evidenced, with $\mathrm{E}_{\mathrm{H}}$ between $19.13 \mathrm{~kJ}$ and 23.30 $\mathrm{kJ}$, depending on the HPMC content in the gels. The obtained gels are thermally and moisture stable, and mucoadhesive on bladder mucosa, which makes them suitable for biologic applications. HPMC/SA blends have been tested for loading and release of two ruthenium(II) complexes, with cytotoxic activity on MEWO cell line, under physiological conditions, thus proving their efficiency in the development of new formulations for drug release systems or carriers based on polysaccharides.

ACKNOWLEDGMENT: This work was supported by a grant of the Ministry of Research and Innovation, CNCS-UEFISCDI, project number PN-III-P1-1.1-PD-2016-1027 (Contract $5 / 2018)$.

\section{REFERENCES}

1 K. Ghosal and S. D. Ray, Braz. J. Pharm. Sci., 47, 4 (2011), http://dx.doi.org/10.1590/S198482502011000400021

2 B. Mirmashhouri, A. D. Tehrani, M. EslamiMoghadam and A. Divsalar, Cellulose Chem. Technol., 52, 27 (2018),

http://www.cellulosechemtechnol.ro/pdf/CCT12(2018)/p.27-33.pdf

3 M. El-Sakhawy, S. Kamel, A. Salama and H.-A. S. Tohamy, Cellulose Chem. Technol., 52, 193 (2018), http://www.cellulosechemtechnol.ro/pdf/CCT34(2018)/p.193-200.pdf

4 S. Rençber, C. N. Cheaburu-Yilmaz, F. A. Köse, S. Y. Karavana and O. Yilmaz, Cellulose Chem. Technol., 53, 655 (2019), https://doi.org/10.35812/CelluloseChemTechnol.2019. 53.64

5 M. El-Sakhawy, A. Salama, S. Kamel and H.-A. S. Tohamy, Cellulose Chem. Technol., 52, 749 (2018),
http://www.cellulosechemtechnol.ro/pdf/CCT910(2018)/p.749-757.pdf

6 Z. Xi, N. Sharma, A. Paprikar and S. Lin, J. Drug Deliv. Sci. Technol., 54, $101231 \quad$ (2019), https://doi.org/10.1016/j.jddst.2019.101231

7 M. Râpa, E. Matei, A. Urcanu, A. M. Predescu, M. C. Pantilimon et al., Cellulose Chem. Technol., 53, 561 (2019), https://doi.org/10.35812/CelluloseChemTechnol.2019. 53.56

8 S. Um-I-Zahra, H. Li and L. Zhu, Cellulose Chem. Technol, $\quad 51, \quad 899 \quad$ (2017), http://www.cellulosechemtechnol.ro/pdf/CCT910(2017)/p.899-909.pdf

9 V. Uivarosi, R. Olar and M. Badea, in "Descriptive Inorganic Chemistry Researches of Metal Compounds", edited by T. Akitsu, Tokyo University of Science, IntechOpen, 2017, http://dx.doi.org/10.5772/intechopen.68306

10 J. Lawless, "The Encyclopedia of Essential Oils", edited by J. Lawless, 2013, pp. 38-39

11 M.-F. Zaltariov, M. Butnaru and D. Peptanariu, in Procs. EHB 2019 Conference, Iasi, November 21-23, 2019

12 M. F. Zaltariov, D. Filip, C.-D. Varganici and D. Macocinschi, Cellulose Chem. Technol., 52, 619 (2018), http://www.cellulosechemtechnol.ro/pdf/CCT78(2018)/p.619-631.pdf

13 G. C. Pimentel and C. H. Sederholm, J. Chem. $\begin{array}{llll}\text { Phys., } & \mathbf{2 4}, & 639 & \text { (1956), }\end{array}$ https://doi.org/10.1063/1.1742588

14 H. Struszczyk, J. Macromol. Sci., 23, 973 (1986), https://doi.org/10.1080/00222338608081105

15 G. Socrates, "Infrared and Raman Characteristic Group Frequencies. Tables and Charts" John Wiley \& Sons, LTD, Chichester, 2001, pp. 76-146

16 A. Mangel, J. Thermal Anal. Calorim., 62, 529 (2000),

https://link.springer.com/article/10.1023/A\%3A101018 3407622

17 L. Zeng, P. Gupta, Y. Chen, E. Wang, L. Ji et al., Chem. Soc. Rev., 46, 5771 (2017), https://doi.org/10.1039/c7cs00195a

18 F. Tas, J. Oncol., ID 647684 (2012), http://dx.doi.org/10.1155/2012/647684 